\title{
ETUDE DES VERS PARASITES DES AMPHIBIENS ANOURES \\ DE LA REGION DE BORDEAUX
}

\author{
NouvelLES ESPĖCES
}

\section{Par J. BAIlenger et J. Chanseau}

L'intensité et l'extrême variété du parasitisme chez les Amphibiens ont de tout temps attiré l'attention des Naturalistes. Ce sont là, en effet, deux facteurs importants pour l'étude taxonomique et biologique des différentes espèces parasitaires.

Il a été ainsi reconnu que le parasitisme s'exerce le plus souvent à un niveau organique précis et s'accompagne d'une spécificité plus ou moins stricte avec mise en jeu de la répartition géographique.

Ainsi, la détermination de la faune parasitaire des Amphibiens d'un pays et même d'une région présente-t-elle un intérêt considérable. Elle doit ètre envisagée en tenant compte, d'une part de la nature des parasites, d'autre part de la fréquence avec laquelle on les rencontre. C'est le double but de ce travail effectué sur les vers parasites des grenouilles de la région de Bordeaux.

\section{1. - Nature des vers parasites des grenouilles de la région de Bordeaux}

La description des Helminthes parasites des Amphibiens est trop classique depuis les travaux de Travassos [12], Walton [13], Karve [7], Lühe [9], Looss [8] pour que nous y revenions. Par ailleurs, une mise au point accompagnée de dessins fera l'objet du travail de l'un de nous (J. C.).

Nous allons seulement opposer les principaux caractères distinctifs et grouper les espèces trouvées en un tableau dichotomique simplifié.

Identifier une espèce, c'est la comparer en tous points à celle qui est classiquement décrite à la suite d'un travail original. Cette identification à la description originale est faite pour chaque ver. Elle nous conduit, pour certains spécimens, à la mise en évidence de

Anv. de Panastrologie, T. XXIX, n 5-6. - 1954 . 
particularités morphologiques et biologiques, voire à l'individualisation d'une nouvelle espèce.

Tels sont les deux aspects de cette étude qui porte sur les vers prélevés à l'autopsie de 119 grenouilles réparties entre Rana esculenta Linné [69], Rana agilis Thomas [11], Rana temporaria Linné [8」 et Hyla arborea var. meridionalis Boettger [31].

Leur étude a été faite en utilisant les méthodes décrites par Bailenger et Neuzil [1].

\section{Principaux caractères et classification des vers parasites des grenouilles de la région de Bordeaux}

Pour établir cette classitication, nous avons choisi les caractères immédiatement apparents, en essayant cependant de ne pas sacrifier à un but pratique les parentés zoologiques que nous respectons, en suivant les grandes lignes de la classification de Ben Dawes [4] pour les Trématodes, de Chitwood [2] pour les Nématodes.

\section{Trématodes}

I. Deux ventouses.

A) Ventouse orale antérieure; ventouse ventrale située à l'extrémité postérieure du corps.

Parasites digestifs.

- 1 testicule au niveau de l'ovaire et s'étendant postérienrement à lui (spécimens adultes); pore génital près de l'orifice buccal; vitellogènes latéraux.

Diplodiscus subclavatus (Pallas, 1760).

- 2 testicules en avant de l'ovaire; pore génital au niveau de la bifurcation intestinale ; vitellogènes occupant postérieurement toute la largeur du corps.

Opisthodiscus diplodiscoides Cohn, 1904.

B) Ventouse orale antérieure; ventouse ventrale en avant de l'extrémité postérieure du corps.

$1^{\circ}$ Poche du cirre; ventouse ventrale plus petite que la ventouse orale; deux testicules; parasites digestifs à l'exception d'Hæmatolæchus.

a) Glandes vitellogènes condensées antérieurement au voisinage de la ventouse orale; pore génital latéral.

a) Ovaire en avant des testicules; poche du cirre affleurant par sa base la ventouse ventrale; 
cæcums courts ne dépassant pas la ventouse ventrale ; ovaire latéral au cæcum droit; testicules de chaque côté de la ventouse ventrale.

Pleurogenoides medians (Olsson, 1876).

ß) Ovaire en arrière des testicules; poche du cirre atteint l'extrémité postérieure du corps.

- Cæcums n'atteignant pas la ventouse ventrale ; ovaire submédian entre le cæcum droit et la ventouse ventrale; testicules de chaque côté de l'œsophage.

Prosotocus fülleborni Travassos, 1930.

b) Glandes vitellogènes étendues dans une grande partie du corps; pore génital submédian; ovaire en avant des testicules.

a) Pore génital dans la zone pharyngienne.

1) Cacums digestifs se terminant au niveau de la ligne médiane du corps; parasites intestinaux.

Cephalogonimus europaeus Blaizot, 1910.

2) Cacums digestifs atteignant l'extrémité postérieure du corps; parasites pulmonaires.

- Cuticule non épineuse; les vitellogènes dépassent postérieurement les testicules.

Haematolochus variegatus (Rudolphi 18:9), Looss, 1899.

— Cuticule épineuse; les vitellogènes atteignent antérieurement les testicules.

Haematoløehus similis Looss, 1899.

ß) Pore génital juste en avant de la ventouse ventrale. Cæcums digestifs atteignant l'extrémité postérieure du corps.

Opisthioglyphe ranae (Frœlich, 1791) Looss, 1907.

$2^{\circ}$ Pas de poche du cirre; ventouse ventrale plus grande que la ventouse orale; 9 testicules; parasites de la vessie.

Gorgodera cygnoides (Zeder, 1800).

II. Une ventouse orale antérieure ; un disque postérieur muni de 6 ventouses et de 2 crochets.

Le tube digestif a des commissures transverses ; les œufs s'accumulent dans l'utérus; papilles vaginales proéminentes; cotylophore grand; 8 à $10 \mathrm{~mm}$. $\times 3$ à $4 \mathrm{~mm}$.

Polystomum integerrimum (Frœlich, 1791) Rudolphi, 1808.

Le tube digestif n'a pas de commissures transverses; les œufs ne s'accumulent pas dans l'utérus ; papilles vaginales rudimentaires ; cotylophore petit; 3,2 à $4 \mathrm{~mm}$. $\times 1$ à $1,4 \mathrm{~mm}$.

Polystomum galliéni Price, 1930. 


\section{Nématodes}

\section{Parasites digestifs.}

A) Absence de bulbe œesophagien, absence de gubernaculum.

$1^{\circ} 3$ lèvres buccales; diverticule intestinal remontant le long de l'œsophage; vulve au tiers antérieur; pas de bourse caudale, spicules égaux courts et épais ; longueur moyenne 7 à $8 \mathrm{~cm}$. (ㅇ), $4 \mathrm{~cm}$. ( ( $)$.

Amplicœeum brumpti Khalil, 1926.

$2^{\circ}$ Manchon céphalique cuticulaire; pas de diverticule intestinal; vulve au tiers postérieur ; bourse caudale; spicules digités; longueur moyenne $1 \mathrm{~cm}$. 5 à $2 \mathrm{~cm}$. (q) ; 0,8 à $1 \mathrm{~cm} .\left(\delta^{\circ}\right)$.

Oswaldocruzia filiformis (Gœze, 1782), Travassos, 1917.

B) Bulbe œsophagien, gubernaculum.

$1^{\circ}$ Extrémité postérieure effilée (ㅇ) ; spicules égaux rudimenmentaires $\left(\delta^{*}\right)$.

10 plectanes préanales constituées par :

un cercle interne finement denticulé, un cercle externe hérissé de 6 à 7 dents.

Cosmocerca ornata (Dujardin, 1845) Raillet et Henry, 1916.

$2^{\circ}$ Extrémité postérieure conique (ㅇ) ; spicules égaux et très longs $(\hat{\delta})$.

Oxysomatium brevicaudatum (Zeder, 1800) Raillet et Henry, 1916.

II. Parasites pulmonaires.

Esophage court.

Rhabdias bufonis (Schrank, 1788) ذ̇tiles et Hassal, 1905.

III. Parasites musculaires.

Papilles et épines céphaliques; queue très courte; vulve antérieure.

Icosiella neglecta (Diesing, 1851) Seurat, 1917.

$3^{\circ}$ Particularités biologiques et morphologiques; nouvelle espèce; nouveau parasite

Parmi les neuf cas de parasitisme par Icosiella neglecta, six s'exerçaient aux dépens de Rana esculenta et deux de Rana temporaria. Cette constatation s'ajoute à celle de de Nabias et Sabrazès [11] et s'oppose à la conception de Desportes [5], pour qui cette 
filaire, de par la biologie de son hòte intermédiaire (Forcipomyia velox Winn., 18522, Diptère Ceratopogonidé), ne se trouve jamais spontanément chez Rana temporaria. Coutelen [3] a d'ailleurs reconnu la possibilité d’infestation expérimentale de cette grenouille.

L'intestin d'une Rana agilis comptait, à còté d'Oswaldocruzia filiformis adultes parfaitement constitués, des Nématodes quatre à cinq fois plus petits, immatures, mais sexuellement différenciés, porteurs notamment de la bourse caudale et des spicules caractéristiques d'Oswaldocruzia filiformis. Leur cuticule, ample, fait des plis; la région céphalique présente plusieurs stades d'une différenciation en manchon ; à l'extrémité postérieure des femelles, une pointe fine et courte, semblable à celle d'Oswaldocruzia filiformis, est nettement individualisée. Il s'agit, nous semble-t-il, de formes « enkystées » constituant un stade évolutif d'Oswaldocruzia filiformis.

Plusieurs Prosotocus retirés de 3 Rana esculenta n'ont pu être identifiés aux dessins, ni aux descriptions de Looss et Travassos, relatifs à l'espèce fülleborni (Travassos, 1930), que nous avons décrite précédemment, et à l'espèce confusus (Looss, 1894).

Il s'agit de petits Trématodes ovoïdes dont les dimensions varient de 0,87 à $1,12 \mathrm{~mm}$. pour la longueur et de 0,47 à $0,58 \mathrm{~mm}$. pour la largeur.

Leur cuticule est hérissée de papilles disposées en rangées régulières. La ventouse orale, subterminale, est un peu plus grande $(0,14$ à $0,19 \times$ 0,13 à $0,17 \mathrm{~mm}$.) que la ventouse ventrale $(0,11$ à $0,14 \times 0,12$ à $0,16 \mathrm{~mm}$.). Cette dernière est située en arrière du milieu du corps.

Le pharynx s'intercale entre le prépharynx et l'œsophage. Celui-ci est court et se bifurque en deux volumineux cæecums inégaux : le gauche, plus petit, ne croise pas la poche du cirre et se termine à quelque distance de la ventouse ventrale.

Les glandes vitellogènes composées d'un petit nombre de volumineux follicules sont condensées au voisinage immédiat de la ventouse orale, en avant des testicules. Ceux-ci constituent deux masses globuleuses $(0,06$ à $0,08 \times 0,11$ à $0,13 \mathrm{~mm}$. $)$ situées latéralement à l'œsophage au niveau de la bifurcation intestinale et antérieurement à l'ovaire. Cette glande, peu lobée, de 0,12 à $0,14 \times 0,09$ à $0,10 \mathrm{~mm}$., est localisée entre le cæcum droit et la ventouse ventrale en position submédiane.

L'utérus longe la face interne de la poche du cirre sur laquelle il ne déborde pas. Antérieurement, il s'étend transversalement entre les glandes vitellogènes et les testicules sous forme d'une bande massive. Il se termine par un vagin musculeux qui s'ouvre au niveau du pore génital, latéralement à gauche, au-dessous des testicules et de la bifurcation intestinale dans la région médiane du corps. A ce niveau s'ouvre aussi 


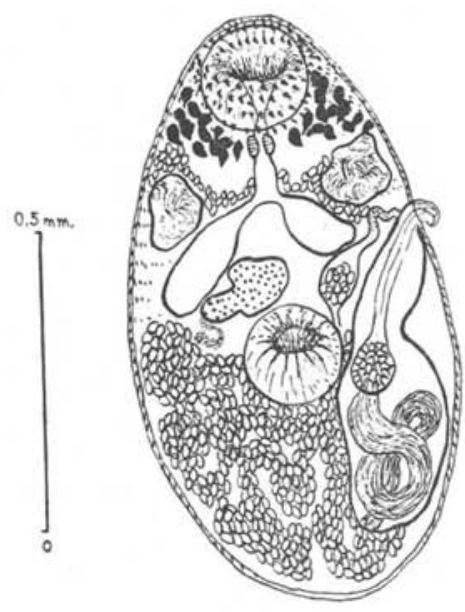

Fic. 1. - Prosotocus fülleborni Travassos, 1930

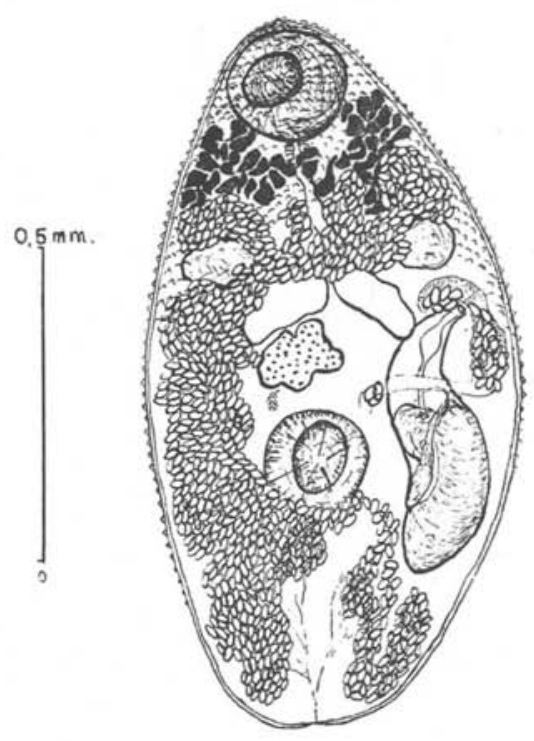

Fic. 2. - Prosotocus sigalasi n. sp.

la poche du cirre. Celle-ci, ample $(0,33$ à $0,41 \times 0,08$ à $0,12 \mathrm{~mm}$. $)$ dépasse postérieurement la ventouse ventrale mais n'atteint pas l'extrémité du corps.

Les œufs, dont la teinte varie du brun clair au brun foncé selon leur degré de maturation, ont des dimensions moyennes de $0,027 \times 0,013 \mathrm{~mm}$. Ils sont piriformes et surmontés d'un petit opercule. 
Les différents caractères d'opposition à Prosotocus fülleborni et confusus, seules espèces connues parasites des Amphibiens, sont résumés dans le tableau suivant. Ils nous conduisent à individualiser une nouvelle espèce que nous dédions à notre Maître, M. le Professeur Sigalas, en hommage de notre respectueuse et profonde gratitude.

\begin{tabular}{|c|c|c|c|}
\hline & $\begin{array}{l}\text { Prosotocus confusus } \\
\text { Looss } 1894\end{array}$ & $\begin{array}{l}\text { Prosotocus } \\
\text { /ülleborni } \\
\text { Travassos 1930 } \\
\quad \text { (figure 1) }\end{array}$ & $\begin{array}{l}\text { Prosotocus } \\
\text { sigalasi } \\
\text { n. sp. } \\
\text { (figure 2) }\end{array}$ \\
\hline Cacum gauche & $\begin{array}{l}\text { Croise la poche du cirre } \\
\text { et atteint la ventouse centrale }\end{array}$ & \multicolumn{2}{|c|}{$\begin{array}{l}\text { Ne croise pas la poche du cirre } \\
\text { et n'atteint pas la ventouse ventrale. }\end{array}$} \\
\hline Pore génital... & $\begin{array}{l}\text { Au niveau de la bifurcation } \\
\text { intestinale dans la zone des } \\
\text { testicules, très en avant du } \\
\text { milieu du corps. }\end{array}$ & \multicolumn{2}{|c|}{$\begin{array}{c}\text { Au dessous de la bifurcation intestinale } \\
\text { et de la zone des testicules, } \\
\text { juste en avant du milicu du corps }\end{array}$} \\
\hline $\begin{array}{l}\text { Branche } \\
\text { utérine } \\
\text { transversale } \\
\text { antérieure }\end{array}$ & $\begin{array}{l}\text { Au niveau des testicules } \\
\text { sans les dépasser antérieu- } \\
\text { rement. }\end{array}$ & $\begin{array}{l}\text { Prétesticulaire } \\
\text { rèduite. }\end{array}$ & $\begin{array}{l}\text { Prélesticulaiıe } \\
\text { massive. }\end{array}$ \\
\hline Poche da cirre & $\begin{array}{l}\text { N'atteint pas l'extrémitè } \\
\text { postérieure du corps. }\end{array}$ & $\begin{array}{l}\text { Atleint l'extrémitè } \\
\text { postérieurducorps }\end{array}$ & $\begin{array}{l}\text { Natteint pas l'ex- } \\
\text { trémité postérieure } \\
\text { du corps. }\end{array}$ \\
\hline Cuticule...... & Epineuse. & $\begin{array}{c}\text { Epineuse } \\
\text { (photographie 5b) }\end{array}$ & $\begin{array}{c}\text { Hérissée } \\
\text { de papilles. } \\
\text { photographie 5a). }\end{array}$ \\
\hline CEufs. ....... & $0,034 \times 0,013 \mathrm{~mm}$. & $\begin{array}{c}\text { Trapus } \\
\text { clapet large } \\
0,025 \times 0,013 \mathrm{~mm} \text {. } \\
\text { (photographie } 5 \mathrm{~d}) .\end{array}$ & $\begin{array}{l}\text { Fusiformes } \\
\text { clapet réduit } \\
0,027 \times 0,013 \mathrm{n}: \mathrm{m} \text {. } \\
\text { (photographie } 5 \mathrm{c} \text { ). }\end{array}$ \\
\hline
\end{tabular}

A côté des différents Trématodes et Nématodes que nous venons de décrire, nous avons isolé de l'intestin d'une Rana esculenta un Acanthocéphale (Fig. 3 et 4 ).

De forme cylindrique, il mesure $5,41 \mathrm{~mm}$. de long sur $0,33 \mathrm{~mm}$. de de large. Sa cuticule ne porte pas d'épines.

Il est plus particulièrement caractérisé par un rostre cylindrique de $0,87 \mathrm{~mm}$. de long (soit un peu plus du sixième de la longueur totale du 


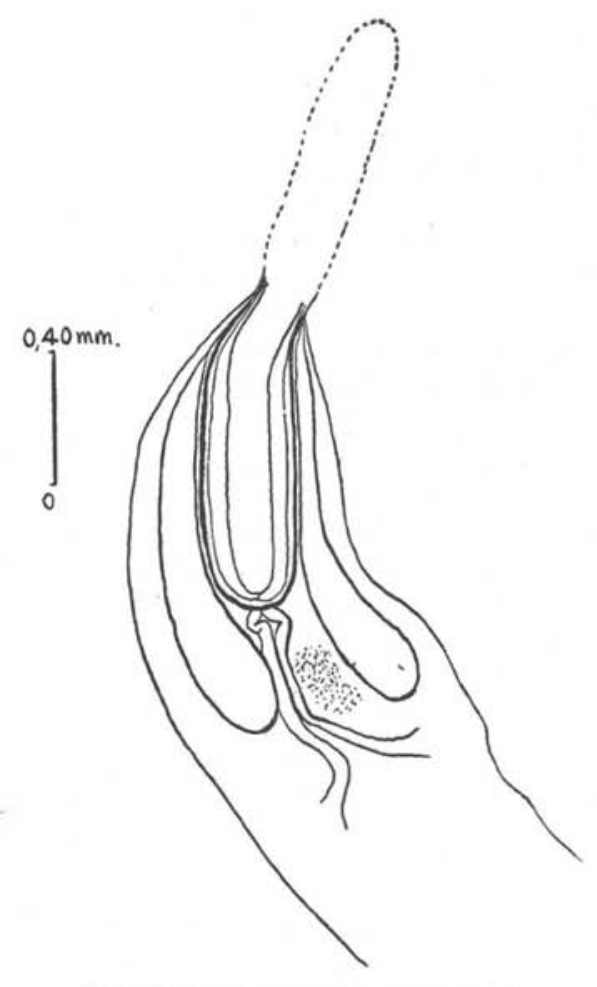

Fig. 3 (ci-dessus)

Fig. 3. - Acasthocéphale isolé de l'intestin de Rana esculenta.

FiG. 4 (ci-contre)

FIG. 4. - Rostre d'un Acanthocéphale isolé de l'intestin de Rana esculenta.

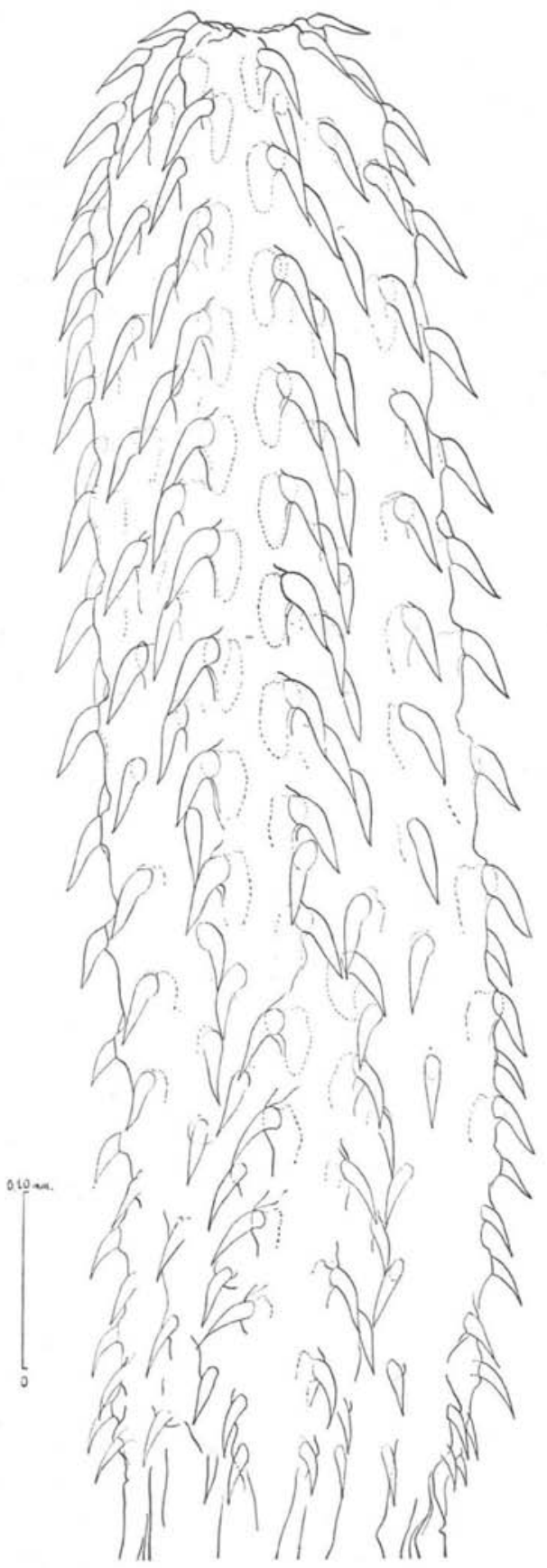


corps), et dont les diamètres de l'extrémité distale, de la région moyenne et de la base mesurent respectivement $0,17-0,18$ et $0,16 \mathrm{~mm}$. Ce rostre est muni de 17 rangées longitudinales régulières de 15 crochets chacune. Schématiquement on peut le diviser en deux parties d'après la constitution des crochets : $1^{\circ}$ une rangée distale comprenant les 10 crochets terminaux caractérisés par leur courbure à angle obtus et par l'existence d'une racine légèrement plus courte qu'eux. Leurs dimensions varient entre $0,041-0,045$ et $0,052 \mathrm{~mm}$; $2^{\circ}$ une région proximale comprenant les 5 crochets de la base. Ils ne sont pas coudés. Leur racine est de moins en moins différenciée et les trois derniers en sont même dépourvus. Ils mesurent de 0,040 à $0,033 \mathrm{~mm}$.

La gaine du rostre s'insère à l'extrémité postérieure de celui-ci. Elle présente une double enveloppe musculaire, d'épaisseur constante, et s'étend sur $1,02 \mathrm{~mm}$. de long et $0,28 \mathrm{~mm}$. de large. Les lemnisques la dépassent de $0,32 \mathrm{~mm}$., soit environ du tiers de sa longueur. De la base de la gaine partent deux ligaments qui se terminent en s'élargissant. En arrière de la gaine, à $0,12 \mathrm{~mm}$. environ, contre les lemnisques, s'individualise une masse d'apparence glandulaire.

Cette description est évidemment incomplète puisqu'elle ne tient pas compte de l'appareil génital que nous n'avons pu individualiser. Peut-être s'agit-il d'une forme immature.

Quoi qu'il en soit, cet Acanthocéphale ne s'apparente pas à Acanthocephalus ranx, parasite classique des Amphibiens anoures. Il est possible d'ailleurs que nous soyons en présence d'un parasite égaré. En ne considérant que le point de vue taxonomique, il est difficile de le classer dans la systématique établie par Lühe [9] et plus récemment par Anton Meyer [10].

\section{II. - Fréquence du parasitisme des grenouilles par les vers}

Cette statistique est établie sur des grenouilles capturées au début du printemps.

Le tableau I ci-après résume l'intensité du parasitisme en fonction de la localisation organique. Le tableau II précise la fréquence des différentes espèces de parasites.

\section{$1^{\circ}$ Nématodes}

Leur fréquence est grande dans le genre Rana où ils sont essentiellement intestinaux et représentés par l'association à peu près constante d'Oxysomatium brevicaudatum et de Cosmocerca ornata. Ces espèces n'ont pas été isolées d'Hyla arborea qui, par contre, ren- 


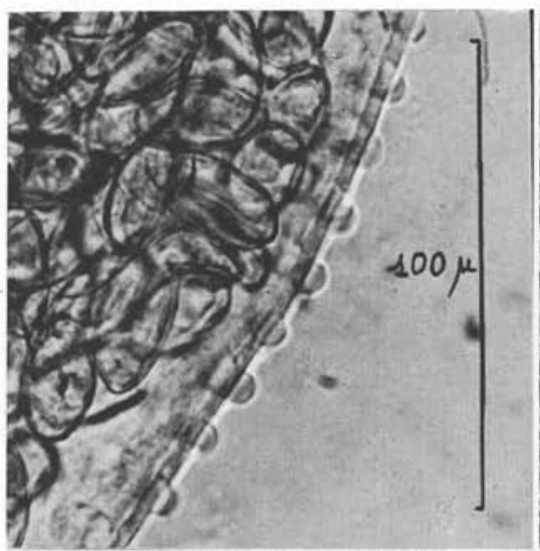

b

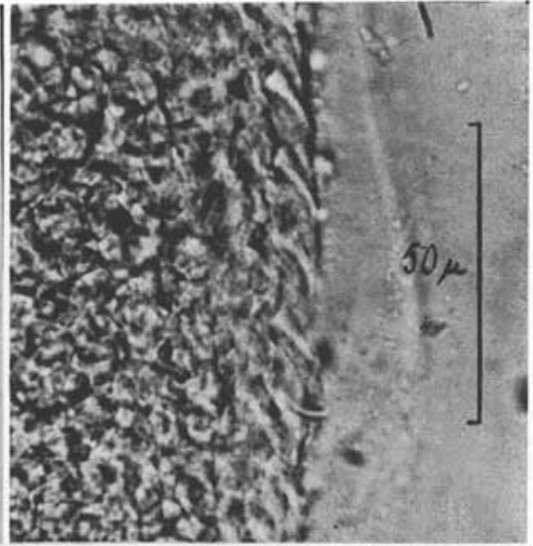

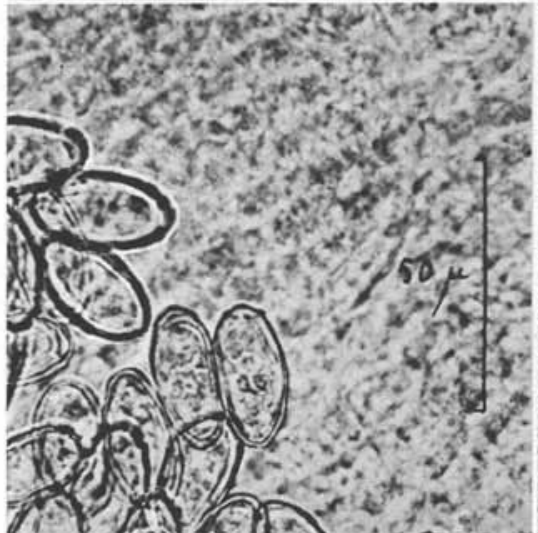

c

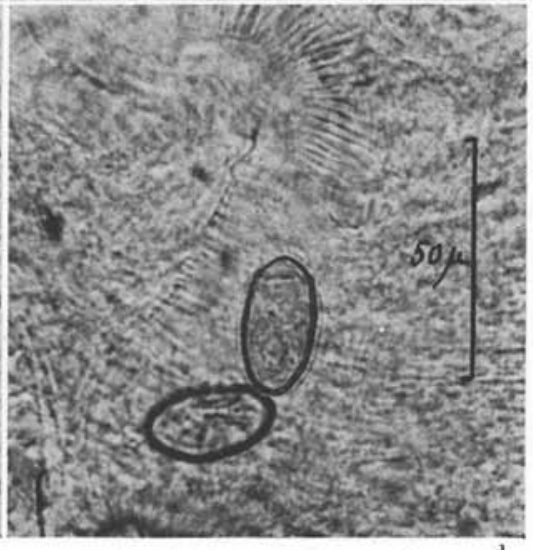

Fig. 5. - Photographies (publiées in Ann. Paras., XXVIII, 1953, p.' 396. J. Bailenger et E. Neuzil, pl. 1, 3, 4, 5, 6) : a) Prosotocus sigalasi n. sp. : cuticule ; b) Prosotocus fülleborni Travassos, 1930 : cuticule ; c) Prosotocus sigalasi : aufs ; d) Prosotocus fïlleborni : cufs.

ferment exclusivement Oswaldocruzia filiformis, également rencontré chez Rana agilis. Les Rana esculenta autopsiées sont porteuses d'Amplicacum brumpti dans la proportion de $4 \%$.

Le parasitisme pulmonaire n'a été étudié que chez Rana esculenta dont le $1 / 4$ environ présente Rhabdias bufonis. Ce Rhabdiasidé n'a pas été trouvé chez Hyla.

Icosiella neglecta parasite à peu près $10 \%$ des Rana esculenta ainsi que deux $R$. temporaria. Cette particularité a fait l'objet d'une discussion antérieure. 


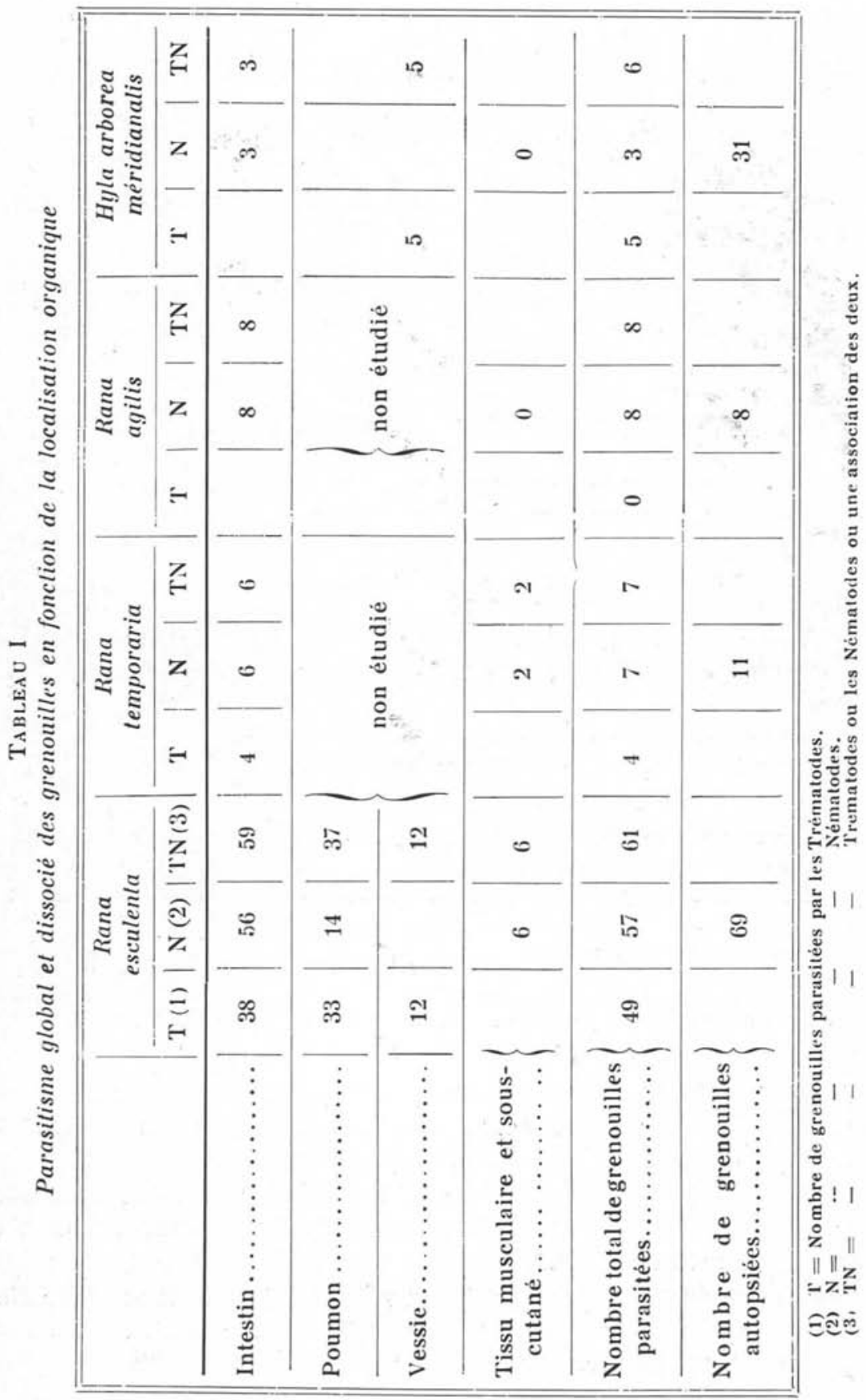


TABLEaU II

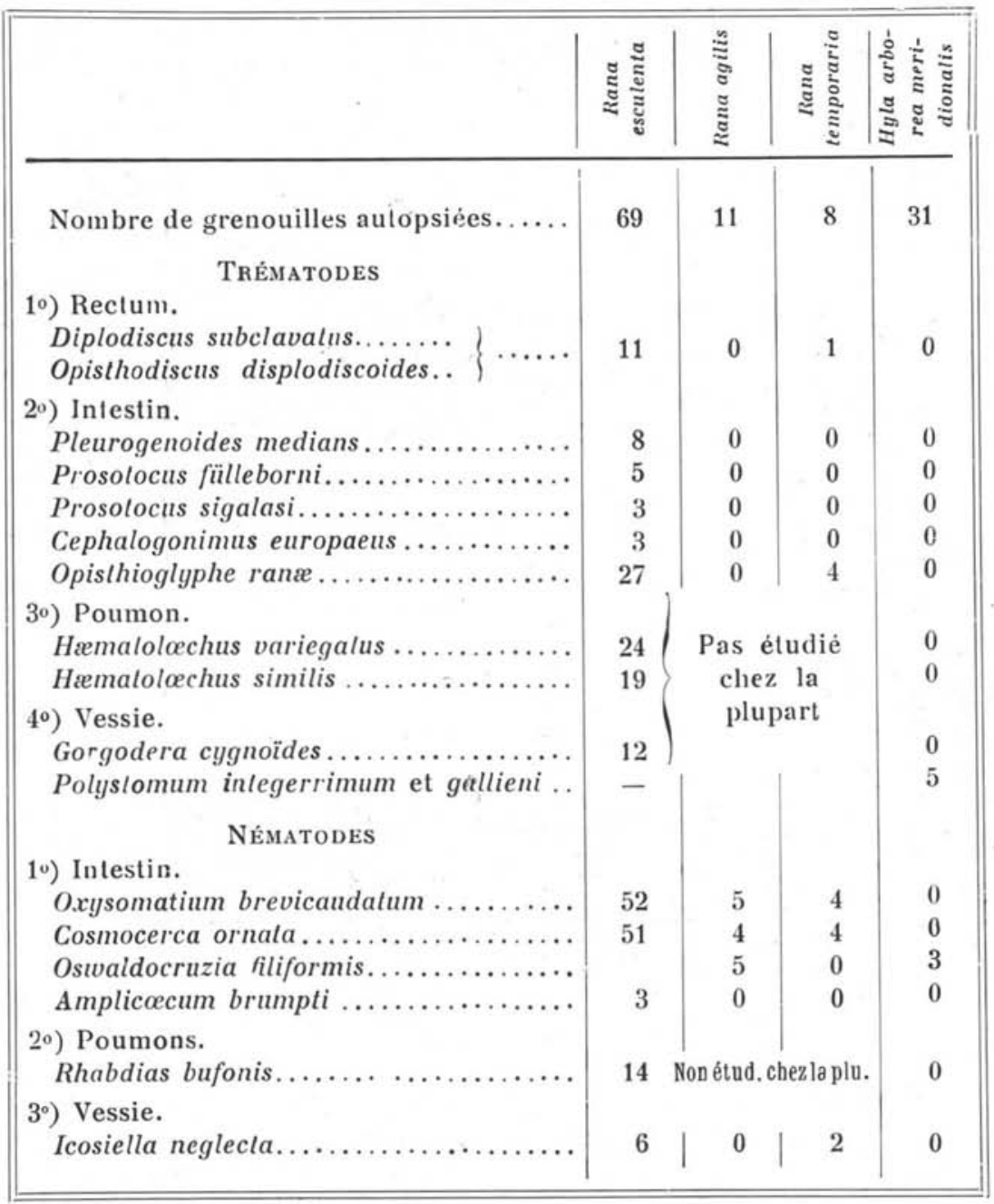

\section{Trématodes}

En comparant la fréquence du parasitisme par les Nématodes et les Trématodes, on note une prédominance des premiers dans l'intestin, des 'Trématodes dans les poumons.

Chez Rana agilis et Hyla arborea, aucun Trématode intestinal 
n'est signalé. Rana esculenta et $R$. temporaria renferment essentiellement Diplodiscus - Opisthodiscus (*). La grenouille rousse n'est parasitée que par les deux douves. La grenouille verte cède en outre à l'autopsie, dans un ordre de fréquence décroissante, Pleurogenoüdes medians, Prosotocus fülleborni, Prosotocus sigalasi, Cephalogonimus europæus.

Au niveau des poumons, Hæmatolæchus variegatus et $H$. similis n'ont été isolés que chez Rana esculenta, et ceci avec une fréquence à peu près égale, ce qui ne signifie pas que ces deux douves soient constamment associées. Leur coexistence, en effet, n'a lieu que dans le $1 / 3$ des grenouilles parasitées.

$17 \%$ des grenouilles vertes renferment dans leur vessie Gorgodera cygnoïdes et $16 \%$ des Hyla sont parasitées par le genre Polystomum.

Nos références bibliographiques nous rapportent une seule statistique semblable à celle-ci. Il s'agit d'un travail de Zakharow [14] effectué en Russie sur 50 Rana esculenta. Parmi les 17 espèces de parasites que cet auteur recueille, 6 seulement sont communes aux nôtres.

Le tableau III fait la comparaison.

TABLEAU III

Zakharofr (Russie) Pcurcentag- local

\begin{tabular}{|c|c|}
\hline Diplodiscus .. & 68 \\
\hline Pleurogenoides medians .... & 26 \\
\hline Opisthioglyphe rana ...... & 8 \\
\hline Hæmatoløchus variegatus ... & 52 \\
\hline Hrmatoløechus similis ..... & 34 \\
\hline Gorgodera cygnoides ....... & 42 \\
\hline
\end{tabular}

\section{Conclusions}

L'étude du parasitisme de 119 grenouilles de la région de Bordeaux nous a conduit à un€ classification simplifiée des Trématodes, Nématodes et Acantocéphales, parasites de ces Batraciens. Le remaniement de cette classification en fonction des modalités parasitaires dans d'autres régions permettrait d'établir une systématique des vers parasites des Amphibiens de France.

(*) Ces deux genres sont réunis dans notre statistique, car nous n'avons fait la différenciation qu'après connaissance de l'important travaii de Dollfus (6), c'est-à-dire à un moment où un grand nombre de grenouilles avaient déjà été autopsiées et où l'identification avait été faite avec Diplodiscus, seul mentionné dans les ouvrages classiques. 
Cette analyse nous a permis d'individualiser un Trématode qui, en raison de ses particularités, peut être apparenté au genre Prosotocus sans cependant pouvoir être rattaché à une espèce connue. Il constitue done, à notre avis, une nouvelle espèce parasite de Rana esculenta: Prosotocus sigalasi. Nous avons également isolé un Acantocéphale que nous r’avons pu identifier à aucun des Acantocéphales parasites classiques des grenouilles. Nous le décrivons sans nous permettre de le classer.

Cette étude se résume en une statistique de la fréquence parasitaire.

\section{BIBLIOGRAPHIE}

1. Bailexger (J.) et Neuzil (E). - Nouvelles techniques d'examen des helminthes : fixation ; coloration ; montage. Ann. Parasitol. hum. et comp., 28, 5-6, 1953, 392-398.

2. Chiтwoon (B. G.) et Chiтwoop (M. B.). - An Introduction to Nematology, section I, Anatomy, Baltimore, 1950, $213 \mathrm{pp}$.

3. Covtei.es (F.). - Contribution à la filariose des grenouilles. Infection et surinfection. Ann. Parasitol. hum. et comp., 6, 1928, 196-199.

4. Dawes Bex. - The Trematoda. With special reference to British and other European Forms, Cambridge, 1946, 644 pp.

5. Despontes (C.). - Nouveiles recherehes sur la morphologie et sur l'évolution d'Icosiella Negiecta (Diesing, 1851). Filaire con:mune de la grencuille verte. Ann. Parasitol. hnm. et comp., 18, n 1-2-3, 1941, 46-66.

6. Dollfus (R.-Ph.) et Chabavd (A.). - Quelques Trématodes, Cestodes et Acanthocéphales. Miscellanea Helminthologica Maroccana, 1-3, 1951, 104-235.

7. Kanve (J. N.). - Some parasitic Nematodes of Frogs and Toads. Ann. Trop. Med. Parasitol., 2', 1930,

8. Looss (A.). - Dic Distomen unserer Fische and Frösche. Biblioth. Zool., 16, 1894, $293 \mathrm{pp}$.

9. LuHe (M.). - Register der Acantocephalen and parasitischen Plattwurmer. Dic Süssmasserfauna, Deutschlands, Heft, 16-19, 1909.

10. Meyer (A.). - Acantocephala. Bronn's Klassen und ordnungen des Tierreichs ; Leipzig, 4 (2), 1933, Buch 2, 582 pp.

11. Nabias (DE) et Sabratí.s. - La filaire du sang des grenouilles. Découverte du mâle. Assoc. franc. pour l'avancement des sciences, Congrès de Pau, 1892 , p. 1 .

12. Travassos (L.). - Trichostrongylidas brasilieras oswaldocru-ia n. gen. Braz. Med., 31 (9), 1917.

Contribuiçoes para o conhecimento da fauna helminthologica brasileira.

XIII. Ensaio monografico da familia. Trichostrongylida, Leiper, 1909. Mem. Inst. Osw. Cruz., 13, 1921, 1.

1I. Famille Echinorhynchida, Hamann, 1892. Sub. fam. Centrorhynchina, Travassos, 1919. Mem. Inst. Osu. Cruz., 19 (1), 1926, 32-125.

- Sobre o genero oxysomatium. Bol. Biol., S.-Paulo, 5, 1927, 20-21.

- Pesquizas helminthologícas realisadas em Hamburgo. 
IV. Notas sobre o genero Opisthioglyphe Looss, 1899, e genero proximos. Mem. Inst. Osw. Cruz., 2h (1), 1930, 1-18.

V. Genero Prosotocus Looss, 1899 (Trematoda: Lecithodendriidæ). Mem. Inst. Osu. Cruz., 2' (2), 1930, 57-61.

I. Genero Hiplometra Looss, 1899. Mem. Inst. Osw. Cruz., 23 (4), 1931, 163-168.

IX. Ensaio monographico da familia Cosmocercidæ, Trav., 1925. Mem. Inst. Osw. Cruz., 25 (3), 1931, 237-298 ; - Genero Pleurogenoides Travassos, 1921 (Trematoda : Lecithodendriidæ). Mem. Inst. Osw. Cruz:, 24 (2), 1930, 63-70 ; - Informacôes sobre o genero Pleurogenes Looss, 1896 (Nematoda (sic) ; Lecithodendriidæ). Mem. Inst. Osun. Cruz., 24 (4), 1930, 251-256.

13. Walton (A. C.). - Studics on some Nematodes of North American Amphibia. II. Cryptobranchida. Journ. Parasit., 17 (1), 1930.

- Note on some larval Nematodes found in frog's. Journ. Parasit., 17 (4), 1931, 228-229.

- The Nematoda as parasites of amphibia. Journ. Parasit., 20, 1933, 1-33.

- Parasites of the Ranidx, XI, XII, XIII. Journ. Parasit., 34, Suppl. 78, 1948, 79-80.

- Parasites of the Ranide, XVIII, XIX, XX. Journ. Parasit., n ${ }^{*} 6$, section 2. Suppl., 1949.

Parasites of the Ranide (Amphibia), XLIV. Trans. Amer. Micr. Soc., 68 (1), 1949.

- Parasites of the Amphibia. Trematoda, I, II, III. Journ. Parasit., 37 (5), section 2. Suppl. octobre 1951.

14. ZакнаRоw. - Ueber dic parasitare Helminthenfauna von Rane esculenta in Dongebiet. Russ. Hydrobiol., Z. 8, 1929, 49-53.

(Laboratoire de zoologie et parasitologie de la Faculté de Médecine et de Pharmacie de Bordeaux

Directeur : Professeur R. Sigal.as) 